\title{
Effects of Deposition Conditions of First InSb Layer on Electrical Properties of n-Type InSb Films Grown With Two-Step Growth Method via InSb Bilayer
}

\section{研究代表者 理工学研究部(工学) 前澤 宏一 非常勤研究員

\section{Background}

Due to its excellent electronic characteristics such as the high electron mobility and the narrow band gap, InSb has received a great deal of attention as a good candidate for infrared detectors, high-speed devices and magnetic sensors. However, the heteroepitaxy of InSb on $\mathrm{Si}$ is difficult to achieve because of the large lattice mismatch of about $19.3 \%$ between them. Previous reports illustrated that the use of an InSb bilayer, prepared by the adsorption of 1 monolayer (ML) Sb onto a surface reconstructed by In atoms, can be a good solution of the lattice mismatch problem. The InSb films synthesized on the InSb bilayer were rotated $30^{\circ}$ with respect to the $\mathrm{Si}(111)$ surface. In this case, the lattice mismatch between $\mathrm{InSb}$ and $\mathrm{Si}$ was nominally reduced to about $3.3 \%$. Some groups reported that the two-step growth method is a successful way of growing highly mismatched systems, such as $\mathrm{InSb} / \mathrm{GaAs}$ and $\mathrm{InSb} / \mathrm{Si}$. This growth method consists of an initial low-temperature InSb layer growth $\left(180-240{ }^{\circ} \mathrm{C}\right)$ and a subsequent high-temperature InSb layer growth $\left(350-440{ }^{\circ} \mathrm{C}\right)$.In our previous reports we showed that the electron mobility values of $\mathrm{InSb}$ films synthesized with two step growth procedure via InSb bi-layer range from 18,000-20,000 $\mathrm{cm}^{2} / V$.s. If we attempt to prepare an InSb film with better electrical properties, it is necessary to improve its crystalline quality. To synthesize an InSb film with a higher crystalline quality and better electrical properties, it is necessary to increase the growth temperature. However, as mentioned above, there are some restrictions for high-temperature growth of an InSb film when we use an InSb bilayer. In our previous study, we prepared an InSb film with a high crystalline quality and good electric properties by gradually increasing the growth temperature of the second InSb layer during deposition. Accordingly, in our current study, we tried to improve the crystalline quality of the first InSb layer, by gradually increasing its growth temperature. Moreover, the thickness of the first InSb layer with a low crystalline quality and poor electric properties decreased compared with that indicated in our previous reports. The microstructure and electrical properties of InSb films were compared with our previous data.

\section{Results}

All the depositions were carried out in an OMICRON molecular beam epitaxy (MBE) chamber. Prior to the growth of InSb films, the InSb bilayer was prepared. The growth of InSb films was then performed by the two step growth method on the InSb bilayer. In our previous experiments, a 30 -nm-thick InSb film was grown on the InSb bilayer at a fixed temperature of $200^{\circ} \mathrm{C}$. This film was named $\mathrm{O}-1$. In our current study, two new films were prepared (N-1 and N-2). In the N-1 film, the first InSb layer with the same thickness as that for the old film (O-1) was prepared at a higher 
growth temperature (higher substrate temperature). In the N-2 film, the thickness of the first InSb layer decreased from 30 to $3 \mathrm{~nm}$ and the growth temperature was the same as that for the N-1 film. To increase the growth temperature of the first InSb layer in the $\mathrm{N}-1$ and N-2 films, this layer was deposited at an initial growth temperature of $200{ }^{\circ} \mathrm{C}$, which was gradually increased to $330{ }^{\circ} \mathrm{C}$ during deposition. The growth rate of InSb films was dominated by the In beam flux. The growth was interrupted by closing the shutter. The second InSb layer was then deposited at a starting growth temperature of $380^{\circ} \mathrm{C}$, which was gradually increased to $440{ }^{\circ} \mathrm{C}$ during deposition.

The RHEED pattern of the O-1 film after the deposition of the first InSb layer showed spotty and ringed pattern indicating the formation of rough surfaces and amorphous like crystals. In contrast, the RHEED patterns of the N-1 and N-2 films after the deposition of the first InSb layer showed a clear $2 \times 2$ periodicity indicating the smooth and well-ordered surface of the grown InSb film. The XRD analysis ( $2 \theta / \omega$ scan) of the InSb films showed that samples were heteroepitaxially grown and had no polycrystalline nature. The FWHM values of the $\operatorname{InSb}(111)$ peaks of the O-1, N-1, and N-2 films are 300, 233, and 118 arcsec, respectively. These results mean that the new InSb films (N-1 and $\mathrm{N}-2$ ) show larger crystal sizes and a higher crystalline quality than the old film (O-1). Moreover, the N-2 film with a thinner first InSb layer shows a smaller FWHM value than the N-1 film, indicating its larger crystal size and higher crystalline quality. TEM and FE-SEM images of the films showed lower defects density of new films compare to the old sample. To evaluate the electrical properties of the films, Hall measurement was performed by using the van der Pauw method. The room temperature electron mobility of the new InSb films (N-1 and N-2) was higher than that of the old film (O-1). The room temperature electron mobility of the N-2 film was 38,000 $\mathrm{cm}^{2} /$ (V.s), which is higher than that of the $1.1 \mu \mathrm{m}$ thick InSb film grown on a Si substrate without any buffer layer. On the other hand, new InSb films (N-1 and N-2) showed lower carrier density values than the old InSb film (O-1).

\section{Conclusion}

The n-type InSb films were prepared with a two-step growth method via an InSb bilayer. The heteroepitaxial growth of InSb films with a high crystalline quality and high electron mobility was achieved by modifying the deposition conditions of the first InSb layer. The deposition conditions of the first InSb layer were optimized by increasing its growth temperature and decreasing its thickness. An InSb film with a high electron mobility of $38000 \mathrm{~cm}^{2} /(\mathrm{V} . \mathrm{s})$ was obtained, implying the high capability of this film for high-speed device applications. Here, we conclude that optimizing the growth conditions of the first InSb layer is effective for improving the crystalline quality and electrical properties of InSb films prepared with a two-step growth method via an InSb bilayer. 